\title{
Spectral-Efficient Model for Multiuser Massive MIMO: Exploiting User Velocity
}

\author{
Thang X. Vu*, Trinh Anh $\mathrm{Vu}^{\dagger}$, Symeon Chatzinotas*, and Bjorn Ottersten* \\ * Interdisciplinary Centre for Security, Reliability and Trust (SnT), \\ University of Luxembourg. Email: \{thang.vu, symeon.chatzinotas, bjorn.ottersten\}@uni.lu \\ $\dagger$ Department of Electronics and Telecommunications, \\ VNU University of Engineering and Technology. Email: vuta@vnu.edu.vn
}

\begin{abstract}
The employment of a massive number of antennas in multiple-input multiple-output systems, known as massive MIMO, has drawn a new horizon for future communications systems to support a very large number of users. However, the actual number of active users in massive MIMO are limited by pilots training via the coherence time of the communication channel which is inversely proportional to the user velocity. The current model applies this coherence time for every user to design multiuser massive MIMO, which might result in a suboptimal solution since the users usually move at different speeds in practice. In this paper, we investigate multiuser massive MIMO by taking into consideration the differences in user velocities. In particular, two multiuser models are proposed to maximize the per-user spectral efficiency and the number of served users, respectively. System capacity of the proposed models is provided in analytical expression. Finally, numerical results demonstrate the advantages of our proposed models compared with the reference model.
\end{abstract}

Index terms - Massive MIMO, channel estimation, multiuser model, channel coherence time.

\section{INTRODUCTION}

Future wireless networks will have to address the rapidly increasing demand for high-rate services because of the proliferation of data-hungry applications and mobile handsets. This calls for new communication technologies with higher spectral- and energy-efficiency. Massive multiple-input multiple-output (MIMO) is one of the promising candidates to achieve these requirements [1]. By equipping with a very large number of antennas, a massive MIMO base station (BS) is capable of transmitting multiple information beams to tens or hundreds of users simultaneously with negligible inter-user interference. Notable advantages of massive MIMO includes green communications, high spectral efficiency (SE) and capacity per area [1].

Numerous research efforts have investigated massive MIMO to analyze performance based on fundamental physical layer properties. In [2], the authors study energy efficiency in massive MIMO uplink and propose a corresponding power control algorithm for both full and imperfect channel state information (CSI). The authors in [3], [4] investigate the interaction between energy efficiency and the number of BS antennas and show that the optimal solution can be achieved via joint resource allocation. In [5], the authors demonstrate that good SE can be achievable in MIMO networks with a not-so-large number of BS antennas. We note that these foreseen advantages of massive MIMO are revealed in the presence of CSI at the BS, which is obtained via pilot training phase. The number of orthogonal pilot sequences can be transmitted simultaneously is bounded by a limited time blocks or coherence time, in which the CSI is static. It is shown in [6] that the system SE is maximized when the number of served users is equal to half of the coherence time measured in channel use (c.u.). The SE for multi-cell massive MIMO can be improved by allowing users in adjacent cells to share some common pilots. In this case, pilot reuse should be intelligently utilized to reduce pilot contamination [7-9].

The above-mentioned works on massive MIMO assume a fixed coherence time applied to all users, which might result in suboptimal solutions since the users usually move with different velocities in practice. In this paper, we investigate the massive MIMO downlink in practical scenarios in which the users' velocities are not identical. The idea of using asymmetric pilots for users with different velocities is introduced in [4]. However, no analysis result on the impact of user velocities is presented. In general, the coherence time is inversely proportional to the relative velocity between transmitter and receiver. Since the BS location is static in our case, the coherence time is determined by the user velocity. Therefore, users moving with a low velocity experience a longer coherence time than users moving with higher velocity. This motivates us to design an adaptive training phase which exploits the nonidentical users' velocities in order to maximize the system SE. In particular, two multiuser models are proposed based on two design criteria which optimize the per-user spectral usage and the number of served users, respectively. The rationale behind our proposed models is to allow users moving with low velocity can share a common pilot signal without interfering the data transmission of users moving with higher speeds. System capacity is provided in analytical expression which reveals significant SE improvement compared with the reference model. Finally, numerical results are presented to demonstrate the effectiveness of our proposed models.

The rest of the paper is organised as follows. Section II describes the system model. Section III proposes a multiuser model to maximize the per-user SE. Section IV proposes a model to maximize the number of served users. Section V provides numerical results. Finally, Section VI concludes the paper.

\section{SYSTEM MODEL}

We consider the massive MIMO downlink channel operating in time-division duplex (TDD) mode, in which a 
users' velocities, which can be effectively obtained at the BS via position and velocity estimating algorithm [11]. Let $v_{\max }$ denote the maximum velocity corresponding to the coherence time $T$. From the Clark model, the relation between these two parameters has the form

$$
v_{\max }=\frac{\Delta}{T}
$$

where $\Delta$ is some constant.

Let $L$ be an integer within the closed interval $[2, K]$. We divide the $K$ users into $L$ groups $\mathcal{U}_{l}, 1 \leq l \leq L$, as follows:

$$
\mathcal{U}_{l}= \begin{cases}\left\{\mathrm{u}_{k} \mid \frac{1}{l+1} v_{\max }<v_{\mathrm{u}_{k}} \leq \frac{1}{l} v_{\max }\right\}, & \text { if } l<L \\ \left\{\mathrm{u}_{k} \mid 0<v_{\mathrm{u}_{k}} \leq \frac{1}{L} v_{\max }\right\}, & \text { if } l=L\end{cases}
$$

where $v_{\mathrm{u}_{k}}$ denotes the velocity of user $\mathrm{u}_{k}$. It is observed that all users in group $\mathcal{U}_{l}$ will experience a coherence time larger than or equal to $T_{l}=l T$, which implies that the channel fading for the users in $\mathcal{U}_{l}$ will be static for a duration of $T_{l}$. Based on this, we propose a novel multiuser model to optimize the per-user SE for users with low velocity, hence improve the system SE. We name this model as Design 1 for convenience. Let $k_{l}=\left|\mathcal{U}_{l}\right|$ denote the number of users in the group $\mathcal{U}_{l}$.

\section{A. Pilot design}

Let $\boldsymbol{\Phi}_{l} \in \mathbb{C}^{k_{l} \times k_{l}}$ be a pilot matrix whose rows are mutually orthogonal and have total energy $\rho$, i.e., $\Phi_{l, i} \Phi_{l, k}^{T}=\rho \delta_{i k}$ and $\left\|\Phi_{l, i}\right\|^{2}=\rho, \forall 1 \leq i \leq k_{l}$, and $\Phi_{l, i}$ is the $i$-th row of $\Phi_{l}$. For each transmission block $t=1,2, \ldots$, define a set $\mathcal{I}(t) \triangleq$ $\{1\} \cup\{k \in\{2, \ldots, L\} \mid t \bmod k=1\}$. As an example, for $L=3$, then $\mathcal{I}(5)=\{1,2\}$.

Given the number of groups $L$, we design pilot signal for the $t$-th block, $\boldsymbol{\Phi}^{1}(t)$ as follows:

$$
\boldsymbol{\Phi}^{1}(t)=\left[\begin{array}{cccc}
\boldsymbol{\Phi}_{i_{1}} & & & 0 \\
& \boldsymbol{\Phi}_{i_{2}} & & \\
& & \ldots & \boldsymbol{\Phi}_{i_{|\mathcal{I}(t)|}}
\end{array}\right],
$$

where $i_{k}$ is the $k$-th element in $\mathcal{I}(t)$.

By definition, $\boldsymbol{\Phi}^{1}(t)$ is of size $\tau_{t} \times \tau_{t}$, where $\tau_{t}=$ $\sum_{l \in \mathcal{I}(t)} k_{l}$, and each row of $\boldsymbol{\Phi}^{1}(t)$ has total energy $\rho$. Since the rows of $\boldsymbol{\Phi}^{1}(t)$ are mutually orthogonal, it can be used to acquire CSI for $\tau_{t}$ users in $\mathcal{U}_{l \in \mathcal{I}(t)}$. It is worthy noting that the pilot design (8) obtains a similar CSI accuracy as the reference model in Section II because the total pilot power in both schemes is $\rho$. It is observed that $\boldsymbol{\Phi}^{1}(t)$ occupies $\tau_{t}$ c.u. and is periodically repeated every $\Gamma T$ c.u., where $\Gamma$ is the least common multiple of $1,2, \ldots, L$. The construction of pilot in Design 1 is demonstrated in Fig $1 \mathrm{~b}$ with $L=3$. In this case, the pilot transmission is periodically repeated every $6 T$ c.u.

Example 1: Let us consider the pilot design for the case $K=4, L=2$ and $k_{1}=k_{2}=2$. In this case, we choose $\boldsymbol{\Phi}_{1}=\boldsymbol{\Phi}_{2}=\frac{1}{\sqrt{2}}[1-1 ; 11]$. The pilot signal of block $t=$ $1,2, \ldots$ is of the form:

$$
\boldsymbol{\Phi}^{1}(t)=\left[\begin{array}{cc}
\frac{1}{\sqrt{2}} & -\frac{1}{\sqrt{2}} \\
\frac{1}{\sqrt{2}} & \frac{1}{\sqrt{2}}
\end{array}\right], \text { if } t \text { is even, }
$$

and

$$
\boldsymbol{\Phi}^{1}(t)=\left[\begin{array}{cccc}
\frac{1}{\sqrt{2}} & -\frac{1}{\sqrt{2}} & 0 & 0 \\
\frac{1}{\sqrt{2}} & \frac{1}{\sqrt{2}} & 0 & 0 \\
0 & 0 & \frac{1}{\sqrt{2}} & -\frac{1}{\sqrt{2}} \\
0 & 0 & \frac{1}{\sqrt{2}} & \frac{1}{\sqrt{2}}
\end{array}\right] \text {, if } t \text { is odd. }
$$

The construction of $\boldsymbol{\Phi}^{1}(t)$ in practical systems can be implemented with the aid of medium access control (MAC) layer. Once obtained the knowledge of users' velocity [11], the BS broadcasts the information about subgroup and block indexes, from which the users determine when to start the training and transmission phases.

\section{B. Data transmission:}

At the beginning of the $t$-th block, the users in $\mathcal{U}_{l \in \mathcal{I}(t)}$ transmit pilot signal $\boldsymbol{\Phi}^{1}(t)$ to the BS for CSI acquisition. The transmission of $\boldsymbol{\Phi}^{1}(t)$ occupies $\tau_{t}$ c.u. After obtaining the CSI, the BS transmits data to all users in the next $T-\tau_{t}$ c.u.

Theorem 1: The system capacity of the proposed multiuser Design 1 is given as

$$
C_{1}=K\left(1-\sum_{l=1}^{L} \frac{k_{l}}{l T}\right) \bar{R} .
$$

Proof: By the design in (8), the pilot signal at the first block is of size $K \times K$. After the training phase, the BS has CSI for all $K$ users. In the transmission phase of the first block, the BS precodes data and then broadcasts it to the $K$ users. The transmission phase in the first block takes place in $(T-K)$ c.u. After the training period of the second block, the BS obtains the updated CSI for $k_{1}$ users in $\mathcal{U}_{1}$. Because the users in groups $\mathcal{U}_{2}, \ldots, \mathcal{U}_{L}$ experience a coherence time larger than $2 T$, the BS uses the CSI obtained in the first block to communicate with these users in the second block. The transmission phase in the second block occupies $T-k_{1}$ c.u. In general, after the training phase of the $t$-th block, the BS obtains the CSI of all users in $\mathcal{U}_{l \in \mathcal{I}(t)}$. We will show that the BS can still transmit data to all $K$ users. For $a, b \in \mathbb{N}^{+}$, define

$$
[a \backslash b]=\left\{\begin{array}{ll}
a \bmod b, & \text { if } a \bmod b>0 \\
b, & \text { if } a \bmod b=0
\end{array} .\right.
$$

Then, for any $l^{\prime}$ with $2 \leq l^{\prime} \leq L$ and $l^{\prime} \notin \mathcal{I}(t)$, the BS uses the CSI obtained in block $t-\left[t \backslash l^{\prime}\right]+1$, because the coherence time for users in group $\mathcal{U}_{l^{\prime}}$ is equal to $l^{\prime} T$, which is absolutely larger than $t \backslash l^{\prime}-1$. It is observed that the process is periodically repeated after $\Gamma$ blocks, where $\Gamma$ is the least common multiple of $1,2, \ldots, L$. Therefore, the system capacity is calculated as follows:

$$
C=\frac{1}{\Gamma} \sum_{t=1}^{\Gamma} K\left(1-\tau_{t}\right) \bar{R}=\frac{1}{\Gamma} K \bar{R}\left(\Gamma-\sum_{t=1}^{\Gamma} \tau_{t}\right),
$$

where $\tau_{t}=\sum_{l \in \mathcal{I}(t)} k_{l}$.

By basic counting method, we observe that the pilot $\Phi_{l}$ for users in $\mathcal{U}_{l}$ is transmitted $\frac{\Gamma}{l}$ times during $\Gamma$ blocks. Thus, we have

$$
\sum_{t=1}^{\Gamma} \tau_{t}=\sum_{l=1}^{L} \frac{\Gamma}{l} k_{l}
$$


Substituting (12) into (11), we obtain the proof of Theorem 1.

Compare (9) with (4) while noting that $\sum_{l=1}^{L} k_{l}=K$, the proposed Design 1 can significantly improves the system capacity by reducing the training phase for users moving with low velocity. Such improvement depends on the number of groups $L$ and how the user number is distributed with respect to (w.r.t.) user velocity (see definition in (13)). In case of uniform distribution, i.e., $p(v)=v K / v_{\max }$, the proposed Design 1 achieves a system capacity

$$
K \bar{R}\left(L-\sum_{l=1}^{L} \frac{l-1}{l}\right) .
$$

As for illustration, with $L=2$ and uniform users distribution and $K=T / 2$, the proposed Design 1 achieves $C^{*}=5 T \bar{R} / 16$, which is $25 \%$ larger than the maximum capacity of the current model in (5). In other words, by dividing users into two groups of different velocities, the system capacity is increased by $25 \%$.

\section{Optimization of users grouping}

This subsection aims at optimizing users' velocity partitions such that the system capacity under Design 1 is maximized. In general, a group of $K$ users can be divided at most into $K$ subgroups each consisting of one user. Denote $p(v)$ as cumulative density function (CDF) of the number of users w.r.t. velocity defined as

$$
p(x)=\left\{\left|\mathcal{U}_{x}\right| \mid v_{\mathrm{u}} \leq x, \forall \mathrm{u} \in \mathcal{U}_{x}\right\}, 0<x \leq v_{\max }
$$

Obviously, $p\left(v_{\max }\right)=K$.

We are interested in selecting a subset $\left\{i_{1}, i_{2}, \ldots, i_{L}\right\} \subset$ $\{1,2, \ldots, K\}$, where $i_{1}=1$ and $i_{1}<i_{2}<\cdots<i_{L}$, to achieve the maximum system capacity. For ease of presentation, denote $i_{L+1}=\infty$. Then the number of users in the $l$-th group is equal

$$
k_{l}=p\left(\frac{v_{\max }}{i_{l}}\right)-p\left(\frac{v_{\max }}{i_{l+1}}\right), 1 \leq l \leq L .
$$

Substituting $k_{l}$ into (9) and after some algebraic manipulations, the optimization of users grouping is formally stated as

$$
\begin{aligned}
\underset{\left\{i_{1}, i_{2}, \ldots, i_{L}\right\}}{\operatorname{maximize}} & K \bar{R}\left(1-\frac{K}{T}+\frac{1}{T} \sum_{l=2}^{L}\left(\frac{1}{i_{l-1}}-\frac{1}{i_{l}}\right) p\left(\frac{v_{\max }}{i_{l}}\right)\right) \\
\text { s.t. } & i_{l} \in\{1, \ldots, K\}, \forall l \\
& i_{1}<i_{2}<\cdots<i_{L} .
\end{aligned}
$$

For arbitrary CDF $p(v)$, solving problem (14) requires searching over $\left(\begin{array}{c}L-1 \\ K-1\end{array}\right)$ possibilities, where $\left(\begin{array}{l}k \\ n\end{array}\right)$ denotes the binomial coefficients. In case of uniform distribution, i.e., $p(v)=v K / v_{\max }$, and $L=2$, the optimization (14) becomes

$$
\underset{i_{2} \in\{1, \ldots, K\}}{\operatorname{maximize}} \frac{K^{2} \bar{R}}{T}\left(\frac{1}{i_{2}}-\frac{1}{i_{2}{ }^{2}}\right),
$$

which has exact closed-form solution $i_{2}=2$. This result shows that in case of $L=2$ and uniform user distribution, it is
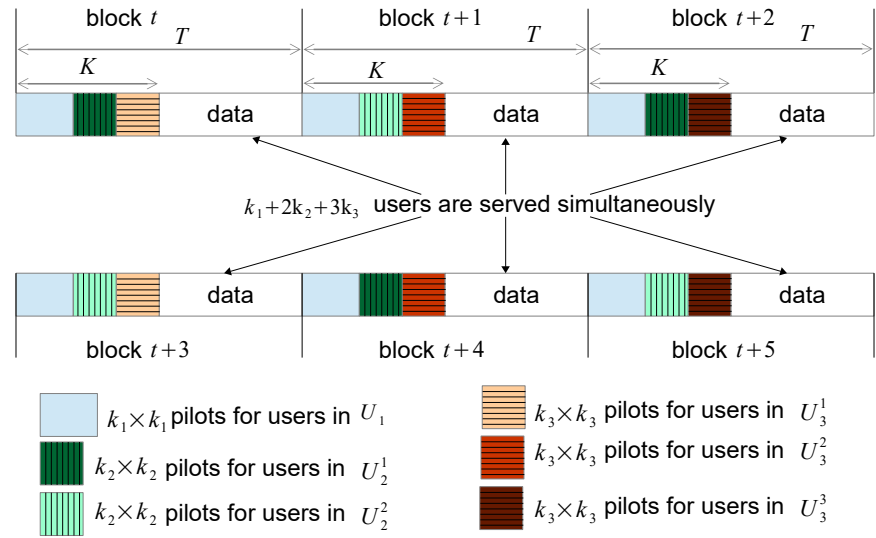

Fig. 2: Proposed multiuser model for maximizing the number of served users. The users are divided into $L=3$ subgroups. The process is repeated periodically every $6 T$ c.u.

optimal to choose a velocity threshold which is half of $v_{\max }$ to maximize the system capacity.

\section{Proposed MultiUser Model: Maximization of NUMBER OF SERVED USERS}

The model proposed in Section III improves the system capacity by allocating more times on the transmission phase for each user. In this section, we propose a novel multiuser design, named Design 2, which can support more active users. The advantage of this design is that it utilizes similar data transmission phase as in the reference model, thus requires a minimum system adjustment. It is assumed in this section that there are many users in the system and that the BS has knowledge on the velocities of all available users [11]. It is worth reminding that the reference model can only serve $K$ users with the coherence time $T$ simultaneously, where $T$ is the system parameter and is usually taken within a few predefined values.

Let $L$ be an arbitrary integer such that $2 \leq L \leq K$. We divide all available users into $L$ groups $\left\{\mathcal{U}_{l}\right\}_{l=1}^{L}$ defined as in (7). Then, let $k_{l}$ with $1 \leq l \leq L$ be the number of active users in $\mathcal{U}_{l}$ which is being served in the reference model. By definition, $K=\sum_{l=1}^{L} k_{l}$.

We further divide the group $\mathcal{U}_{l}$ into non-joint subgroups of equal size $k_{l}$ as $\mathcal{U}_{l}=\mathcal{U}_{l}^{1} \cup \mathcal{U}_{l}^{2} \cup \cdots \cup \mathcal{U}_{l}^{\left\lfloor\frac{\left.\mid \mathcal{U}_{l}\right\rfloor}{k_{l}}\right\rfloor}$, where $\left|\mathcal{U}_{l}^{i}\right|=k_{l}, \forall i$, and $\left|\mathcal{U}_{l}^{i} \cap \mathcal{U}_{l}^{j}\right|=\delta_{i j} k_{l}$.

\section{A. Pilot design}

Denote $\boldsymbol{\Phi}_{\mathcal{U}_{l}^{i}} \in \mathbb{C}^{k_{l} \times k_{l}}$ as the pilot signal for the users in $\mathcal{U}_{l}^{i}$, whose rows are mutually orthogonal and have energy $\rho$. We construct the pilot signals of size $K \times K$ for the $t$-th block as follows:

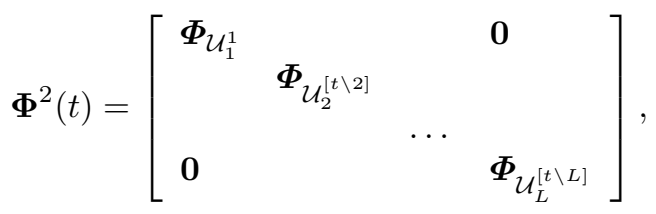

where $[a \backslash b]$ is defined in (10). 
Since the construction of the pilot in (16) satisfies the mutually orthogonal among the rows, it can be used to obtain the CSI for $K$ users simultaneously. Since the total energy of every pilot is $\rho$, (16) achieves a similar CSI accuracy as in Design 1 and the reference model. Illustrated pilots in Design 2 is depicted in Figure 2.

Remark 2: In Design 2, users in different subgroups are allowed to use the same pilot without causing pilot contamination because their training phase are accomplished in different times.

\section{B. Data transmission}

After CSI estimation, the BS transmits precoded data to the users. In Design 2, the training phase takes place in $K$ c.u. and the transmission phase lasts in $T-K$ c.u.

Theorem 2: The system capacity under the Design 2 is calculated as

$$
C_{2}=\left(\sum_{l=1}^{L} l k_{l}\right)\left(1-\frac{K}{T}\right) \bar{R} .
$$

Proof: We start with the first block. After $K$ c.u., the BS obtains the CSI for $K$ users in $\mathcal{U}_{1}^{(1)}, \mathcal{U}_{2}^{(1)}, \ldots, \mathcal{U}_{L}^{(1)}$. In the next $T-K$ c.u., the BS transmits the precoded data to these $K$ users. After the training phase of block two, the BS updates the CSI for users in $\mathcal{U}_{1}^{(1)}$ and obtains the CSI for users in $\mathcal{U}_{2}^{(2)}, \mathcal{U}_{3}^{(2)}, \ldots, \mathcal{U}_{L}^{(2)}$. Because the users in $\mathcal{U}_{l \geq 2}$ experience a coherence time larger than $2 T$, the BS knows the CSI for users in $\mathcal{U}_{2}^{(1)}, \mathcal{U}_{2}^{(1)}, \ldots, \mathcal{U}_{L}^{(1)}$ which was obtained in the first block. Therefore, in the data transmission phase in block two, the BS send precoded data to the users in $\mathcal{U}_{1}^{(1)}, \mathcal{U}_{2}^{(1)}, \mathcal{U}_{2}^{(2)}, \ldots, \mathcal{U}_{L}^{(1)}, \mathcal{U}_{L}^{(2)}$. In general, after the training phase at the $t$-th block, $t \geq L$, the BS has CSI knowledge of all users in $l$ disjoint subgroups $\mathcal{U}_{l}^{(1)}, \ldots, \mathcal{U}_{l}^{(l)}$ in group $\mathcal{U}_{l}$, in which the CSI of users in $\mathcal{U}_{l}^{(i)}$ is obtained in the block $t-t_{i}$, where $t_{i}$ satisfies $0 \leq t_{i}<L$ and $i=\left(t-t_{i}\right) \bmod l$. In the next $T-K$ c.u. in the $t$-th block, the BS transmits precoded data to all these users. The total number of served users is given by $\sum_{l=1}^{L} l k_{l}$. Because the BS only uses $T-K$ c.u. in each block to transmit the data, the system capacity in this case is calculated as

$$
\frac{1}{T}\left(\sum_{l=1}^{L} l k_{l}\right)(T-K) \bar{R}=\left(\sum_{l=1}^{L} l k_{l}\right)\left(1-\frac{K}{T}\right) \bar{R}
$$

It is shown from (17) and (4) that the Design 2 significantly improves the system capacity by allowing more users to share some common pilots. When the number of users has uniform distribution w.r.t. velocity, the system capacity under Design 2 is given as

$$
C=K\left(L-\sum_{l=1}^{L} \frac{l}{l+1}\right)\left(1-\frac{K}{T}\right) \bar{R}
$$

\section{NumericAl RESUlts}

This section presents numerical results of the proposed multiuser designs for a massive MIMO system. The proposed

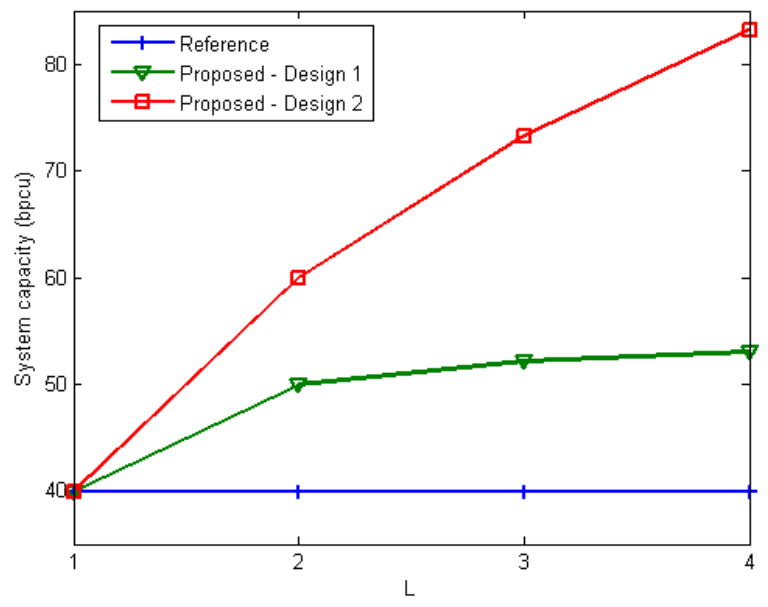

Fig. 3: System capacity comparison of the three models for different number of subgroups. $\mathrm{SNR}=20 \mathrm{~dB}, K=12$.

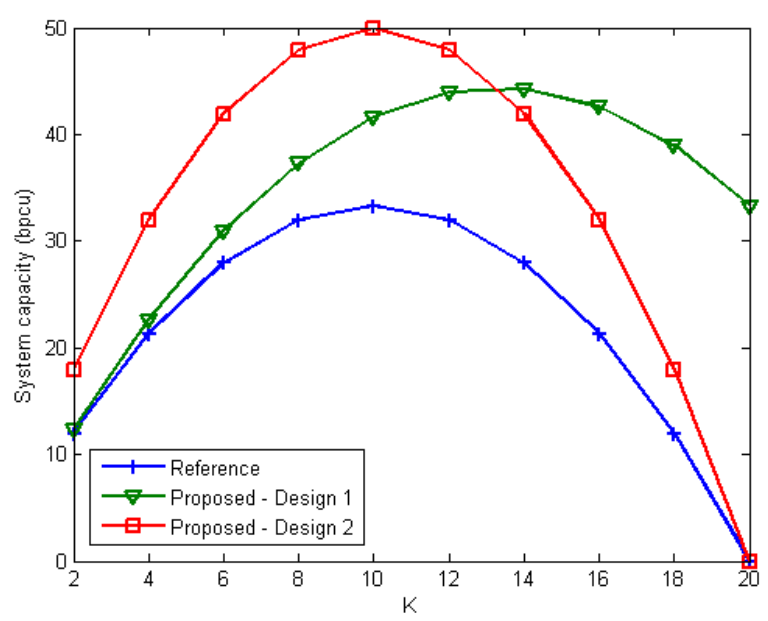

Fig. 4: System capacity comparison of the three models for different $K$. SNR $=20 \mathrm{~dB}, L=2, T=20$ c.u.

designs are compared with the reference model in [6]. Numerical result is obtained for 1000 channel realizations. The number of $\mathrm{BS}$ antennas is $M=200$. Zero-forcing precoder is used [4]. SNR is defined as $\rho / \sigma^{2}$, which is the same for pilot and data. Unless stated otherwise, the user number is uniformly distributed w.r.t. user velocity. Figure 3 shows the system capacity as a function of the group number $L$ at $\mathrm{SNR}=20 \mathrm{~dB}$. Since the reference model treats all $K$ users with the same coherence time, its system capacity is not effected by $L$. It is shown that the proposed models significantly improve the system capacity. In particular, with $L=3$ groups, the proposed Designs 1 and 2 boost the system capacity by $25 \%$ and $85 \%$, respectively. As $L$ increases, larger performance gain is achieved by Design 2. On the other hand, Design 1 slightly improves the system capacity. From the implementation point of view, $L=2$ is good for Design 1 to balance between the performance gain and complexity.

Figure 4 compares the system capacity of three models for 


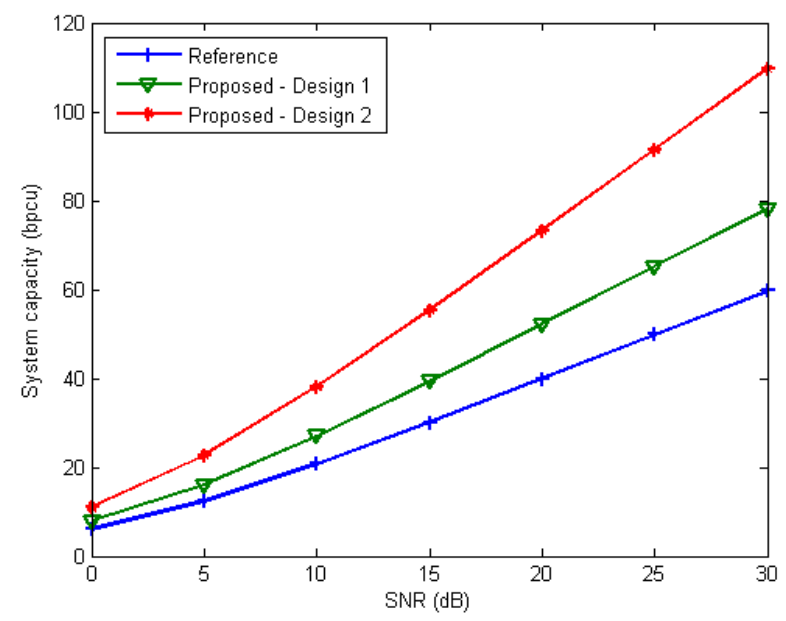

Fig. 5: Performance comparison of the three models for different SNR. $K=12, L=3$.

various $K$. The number of subgroups and the coherence time are fixed at $L=2$ and $T=20$ c.u., respectively. It is observed that the proposed models always outperform the reference model. When $K$ increases but less than $T / 2$, all three models achieve larger system capacity. However, the system capacity of the reference and Design 2 decreases as $K$ exceeds $T / 2$ because in this case the training phase takes more times and therefore, there is less time for data transmission. It is also observed that the Design 2 and reference model achieve the maximum capacity at $K=T / 2$, which can be explained from Theorem 2 as following. The capacity of Design 2 when $L=2$ is equal to $\frac{3}{2} \bar{R} K\left(1-\frac{K}{T}\right) \leq \frac{3}{8} \bar{R} T$, where the equality holds when $K=T / 2$. The Design 2 performs well for $K<T / 2$, while the Design 1 is preferred for $K>T / 2$. Especially, when $K=T$, the reference model and Design 2 can not convey data because there is no time frame for data transmission. However, the proposed Design 1 can still achieve a system capacity of 33 bpcu.

Figure 5 presents the system capacity of three models as a function of SNR for $L=3$. Significant gain is also observed by our models compared with the reference design and this gain is proportional to the SNR, as shown in Theorems 1 and 2. Figure 6 presents the optimal grouping of Design 1 for different user distributions. The non-uniform distribution assumes that there are more users moving with low speed than users moving with high speed, which is reasonable in practice. It is shown that the optimal grouping is more effective for nonuniform distribution, which can be explained from Theorem 1.

\section{CONCLUSIONS}

We proposed two spectral-efficient multiuser models for massive MIMO systems. Our models take into consideration non-identical users' velocities to optimally design pilot and data transmission phases. Two design criteria were proposed to maximize the per-user spectral efficiency or the number of active users, which eventually improve the system capacity. For each design, the pilot structure was proposed and the corresponding system capacity was analysed. Finally, we

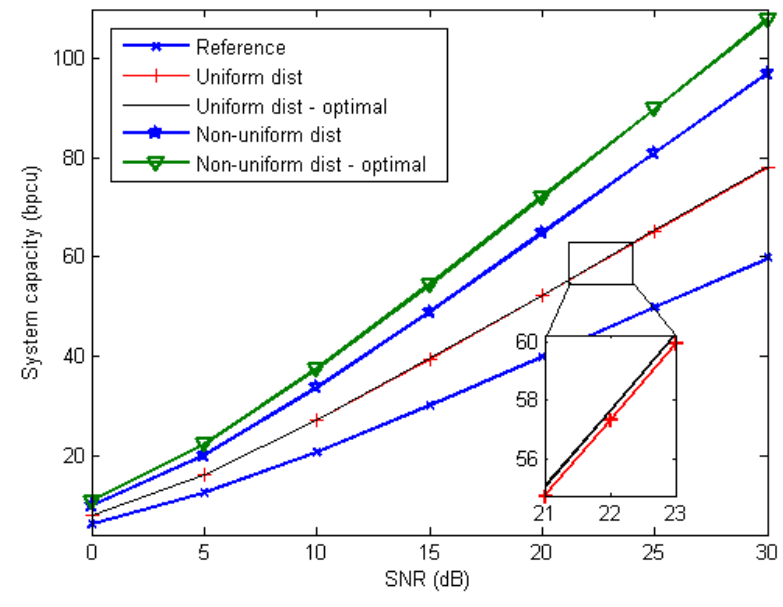

Fig. 6: Effectiveness of optimal grouping in Design 1 for different user distributions. $K=12, L=3$. Uniform distribution: $p(x)=x K / v_{\max }$; non-uniform distribution: $p(x)=$ $\ln \left(\frac{x\left(e^{K}-1\right)}{v_{\max }}+1\right)$.

demonstrated the advantages of our models via numerical results, which showed that significant capacity gain is achieved over the reference model.

\section{ACKNOWLEDGEMENT}

This work is financially supported in part by Luxembourg National Research Fund Core program under the project code I2R-SIG-PFN-13SEMI.

\section{REFERENCES}

[1] E. G. Larsson, O. Edfors, F. Tufvesson, and T. L. Marzetta, "Massive MIMO for next generation wireless systems," IEEE Commun. Mag., vol. 52, no. 2, pp. 186-195, Feb. 2014.

[2] H. Q. Ngo, E. G. Larsson, and T. L. Marzetta, "Energy and spectral efficiency of very large multiuser MIMO systems," IEEE Trans. Commun., vol. 61, no. 4, pp. 1436-1449, Apr. 2013.

[3] J. Hoydis, S. ten Brink, and M. Debbah, "Massive MIMO in the UL/DL of cellular networks: How many antennas do we need?" IEEE J. Sel. Areas Commun., vol. 31, no. 2, pp. 160-171, Feb. 2013.

[4] E. Bjornson, E. G. Larsson, and M. Debbah, "Massive MIMO for maximal spectral efficiency: How many users and pilots should be allocated?" IEEE Trans. Wireless Commun., vol. 15, no. 2, pp. 1293 1308, Feb. 2016.

[5] H. Huh, G. Caire, H. C. Papadopoulos, and S. A. Ramprashad, "Achieving "massive MIMO" spectral efficiency with a not-so-large number of antennas," IEEE Trans. Wireless Commun., vol. 11, no. 9, pp. 32263239, Sept. 2012.

[6] T. L. Marzetta, "How much training is required for multiuser MIMO?" in Proc. Asilomar Conf. Signals Syst. Comput., Oct. 2006, pp. 359-363.

[7] J. Jose, A. Ashikhmin, T. L. Marzetta, and S. Vishwanath, "Pilot contamination problem in multi-cell tdd systems," in Proc. IEEE Int. Symp. Inf. Theory, June 2009, pp. 2184-2188.

[8] H. Yin, D. Gesbert, M. Filippou, and Y. Liu, "A coordinated approach to channel estimation in large-scale multiple-antenna systems," IEEE J. Sel. Areas Commun., vol. 31, no. 2, pp. 264-273, Feb. 2013.

[9] T. X. Vu, T. A. Vu, and T. Q. S. Quek, "Successive pilot contamination elimination in multiantenna multicell networks," IEEE Wireless Commun. Lett., vol. 3, no. 6, pp. 617-620, Dec. 2014.

[10] E. Bjornson, L. Sanguinetti, J. Hoydis, and M. Debbah, "Designing multi-user MIMO for energy efficiency: When is massive MIMO the answer?" in Proc. IEEE Wireless Commun. Netw. Conf., Apr. 2014, pp. 242-247.

[11] M. Hellebrandt, R. Mathar, and M. Scheibenbogen, "Estimating position and velocity of mobiles in a cellular radio network," IEEE Trans. Veh. Technol., vol. 46, no. 1, pp. 65-71, Feb. 1997. 